\title{
Reflets
}

Revue ontaroise d'intervention sociale et communautaire

\section{Le RIFSSSO - un modèle de coopération}

\section{Diane Plante}

Volume 5, numéro 2, automne 1999

La santé des francophones de l’Ontario

URI : https://id.erudit.org/iderudit/026288ar

DOI : https://doi.org/10.7202/026288ar

Aller au sommaire du numéro

Éditeur(s)

Reflets : Revue ontaroise d'intervention sociale et communautaire

ISSN

1203-4576 (imprimé)

1712-8498 (numérique)

Découvrir la revue

Citer cet article

Plante, D. (1999). Le RIFSSSO — un modèle de coopération. Reflets, 5(2),

279-284. https://doi.org/10.7202/026288ar

Tous droits réservés (C) Reflets : Revue ontaroise d'intervention sociale et communautaire, 1999

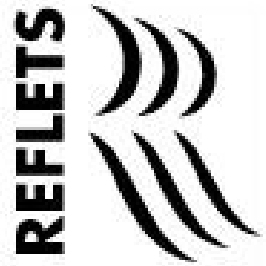

Ce document est protégé par la loi sur le droit d'auteur. L'utilisation des services d'Érudit (y compris la reproduction) est assujettie à sa politique d'utilisation que vous pouvez consulter en ligne.

https://apropos.erudit.org/fr/usagers/politique-dutilisation/ 


\title{
Le RIFSSSO - un modèle de coopération
}

\author{
D iane Plante
}

\section{Les origines du RIFSSSO}

D éjà, plus de dix ans se sont écoulés depuis que les professionnels francophones en santé et en services sociaux ont décidé de se pencher sur la question de la prestation des services en français. Comme ils n'étaient pas suffisamment nombreux pour former des associations disciplinaires francophones distinctes et qu'ils sentaient le besoin de se regrouper, ils ont choisi de mettre sur pied une seule association provinciale représentant l'ensemble de leurs professions. II s'agit là d'un modèle de coopération et de partenariat unique dans le domaine de la santé et des services sociaux au C anada.

Le R egroupement desintervenanteset intervenants francophones en santé et en services sociaux de l'O ntario (R IFSSSO) a donc été créé en 1990. O mer Deslauriers en a été le président fondateur. Jocelyne Lalonde lui a succédé pendant deux ans. Depuis, Diane Plante assume ses fonctions pour la troisième année. Pour sa part, Claudia Lebeuf occupe le poste de directrice générale depuis 1991.

Le R IFSSSO est composé de personnes et de regroupements professionnelsfrancophones œuvrant dansles domaines de la santé et des services sociaux. Les douze regroupements membres du R IFSSSO sont les ambulanciers et ambulancières, les audiologistes et orthophonistes, les dentistes, les diététistes, les éducateurs et éducatrices des services à l'enfance, les ergothérapeutes, les 
hygiénistes dentaires, les infirmières et infirmiers, les physiothérapeutes, les psychologues, les techniciennes et techniciensen éducation spécialisée, les travailleuses et travailleurs sociaux. La liste ne s'arrête pas là puisque plusieurs autres professions sont représentées au sein du R IFSSSO.

Le R IFSSSO vise à promouvoir la communication entre les membres et à favoriser l'amélioration de la prestation des services en français en 0 ntario. Financé par une entente fédérale et provinciale entre Patrimoine canadien et les ministères de la Santé et desServices sociaux et communautaires de l'O ntario, le R IFSSSO travaille aujourd'hui à la mise sur pied d'une entreprise, R IFSSSO PLU S qui remplacera d'ici cinq ans, le financement gouvernemental.

\section{Une philosophie d'économie de moyens}

Au R IFSSSO, le personnel permanent comprend la directrice générale, assistée d'une adjointe administrative. De plus, une analyste et communicatrice assume des responsabilités dans ces domaines à temps partiel. C'est donc dire que les ressources humaines sont plutôt limitées. Lesactivités du R IFSSSO touchent les domaines suivants.

D éveloppement organisationnel: depuis 1991, le R IFSSSO a créé une banque de données de 15000 noms et mis sur pied douze regroupements professionnels. II compte à son actif près de 5000 membres. Le développement organisationnel comprend les activités suivantes: la tenue, au moyen de l'audioconférence ou de la vidéoconférence, de rencontres (soixante au cours de la dernière année), d'ateliers ou de sessions d'informations pour les regroupements. Le RIFSSSO aide chaque regroupement dans I'identification de ses besoins et tâche d'y répondre en offrant une gamme de services variés.

$D$ ans le but de briser l'isolement et de resserrer les liens entre les intervenants, le R IFSSSO tient tous les ans un congrès. D ans le passé, les congrès se sont déroulés sous les thèmes suivants: 
Partenaires dans l'action (1993), C onstruire ensemble l'avenir : le leadership et la force créatrice (1994), U n engagement individuel, une force collective (1995), U ne force collective: au-delà des compressions (1996), B ien être pour bien intervenir (1997), N ouvel environnement, N ouveaux défis! (1998).

C ommunication : le R IFSSSO a produit divers documents dont un bulletin appelé $L$ e R egroupement, publié une ou deux fois par an. De plus, on a publié des bulletins ponctuels qui ont été distribués aux 5000 membres, comme Le R egroupement en bref et Q uoi de neuf? Final ement, presque toutes les associations sublient un bulletin, trois ou quatre fois par année, distribué à leurs membres.

En partenariat avec le ministère de la Santé, le R IFSSSO a lancé la campagne J'me lanceen santé qui comprenait la production d'une vidéo et la publication d'une vingtaine de dépliants sur une variété de carrièresen santé et en servicessociaux. C esderniers ont été distribués dans les écoles françaises de l'O ntario. A ujourd'hui, le R IFSSSO poursuit ce programme en présentant sur Internet la page web: C arrière-0- matique, avec 25 professions, que l'on peut consulter au site R IFSSSO.

Interventions: le R IFSSSO n'est pas un organisme politique. II fait cependant des interventions auprès des instances gouvernementales pour les sensibiliser à certaines décisions qui ont un impact sur l'épanouissement de la communauté francophone. Entre autres, le R IFSSSO afait de nombreuses interventionsauprès de la Commission de la restructuration des services de santé au sujet des services de santé en français dans la région d'O ttawa$C$ arleton, particulièrement sur l'importance pour lesfrancophones de garder ouvert I'H ôpital M ontfort. Le R IFSSSO a également fait des représentations auprès du ministère des Services sociaux et communautaires, au sujet descompressions budgétai res, et auprès du ministère desAffairesmunicipales et du Logement au sujet du transfert de pouvoirs aux municipalités qui ne sont pas régies par la $L$ oi sur les services en français.

R elations extérieures: le R IFSSSO a établi des liens étroits et de nombreux partenariats avec les organismes communautaires, les universités et les collèges communautaires, plus spécifiquement 
avec les directeurs de programmes en santé et en services sociaux, la faculté des sciences de la santé et la faculté de médecine de I'U niversité d'O ttawa, la clinique d'orthophonie de l'U niversité Laurentienne - toujours dans le but de répondre aux besoins des professionnels de la santé et des services sociaux: formation continue, stages, etc.

Dernièrement, le R IFSSSO annonçait le lancement d'un projet de coordination et de prestation de services aux enfants francophones du Sud de I'O ntario, intitulé SEFS. Le projet a pour objectif de permettre aux enfants du Sud de l'O ntario d'avoir accès à des services cliniques offerts sur une base individuelle, familiale ou de groupe. Au besoin, on pourra déborder ce cadre et offrir des services plus préventifs, éducatifs ou communautaires.

Le R IFSSSO collabore régulièrement avec les ministères de la Santé et des Services sociaux et communautaires ainsi qu'avec I'O ffice des affaires francophones. En 1998, il a créé le R éseauN at, un réseau national desintervenantes et intervenants francophones en santé et en services sociaux.

\section{L'importance du RIFSSSO}

Le R IFSSSO est le seul organisme de la province rassemblant les professionnels de la santé et des services sociaux francophones. II a réussi à briser l'isolement dans lequel se trouvai ent les intervenants et intervenants travaillant en santé et en services sociaux, dans un milieu où ilsse trouvai ent en minorité du point de vue linguistique. À leur tour, les intervenants, grâce àl'échange entre professionnels, ont pu améliorer la prestation des services en français, ce qui a contribué au mieux-être des francophones en 0 ntario.

G râce au R IFSSSO, les intervenantes et les intervenants en santé et en services sociaux:

- obtiennent désormais plus de services en français de la part des 0 rdres;

- suivent des ateliers de formation en français; 
- interviennent de façon organisée dans les dossiers de l'heure.

En ce qui concerne le gouvernement de l'O ntario, celui-ci :

- a accès à une banque d'information sur les intervenantes et intervenants en santé et en services sociaux;

- est en mesure de mieux administrer la Loi de 1986 sur les services en français puisqu'il comprend mieux les besoins des francophones en santé et en services sociaux;

- a un partenaire communautaire qui le renseigne et l'aide à offrir ses programmes de santé et de services sociaux;

- peut communiquer à peu de frais, avec les professionnelles et les professionnels de la santé et des services sociaux par l'intermédiaire d'une seule association.

Par ailleurs, le gouvernement du $\mathrm{C}$ anada a un interlocuteur en O ntario, capable et désireux de partager ses expériences avec d'autres provinces. $0 \mathrm{n}$ pense ici au R éseauN at.

\section{Une nouvelle initiative}

En raison de la difficulté de la part des employeurs à recruter du personnel bilingue en santé et en services sociaux, les membres du R IFSSSO ont décidé de mettre sur pied une entreprise qui mettra à profit leur expertise en tant que professionnels bilingues travaillant dans les domaines de la santé et des services sociaux. Le lancement de l'entreprise, enregistrée sous le nom de R IFSSSO -PLU S est prévu pour l'automne 1999.

R IFSSSO - PLU S sera un centre de services professionnels unique au $\mathrm{C}$ anada puisqu'il sera francophone et bilingue. $\mathrm{Ce}$ service sera basé sur la banque de données du RIFSSSO qui comprend 15000 noms. R IFSSSO -PLU S sera en mesure de fournir des services diversifiés et personnalisés de la plus haute qualité dans le milieu.

Les composantes de R IFSSSO - PLU S seront les suivantes:

- service de placement à plein temps;

- service de placement à temps partiel; 
- service de placement en utilisant Internet;

- service de formation professionnelle;

- services connexes recherche, analyse, coordination de projets, sondages, etc.

Finalement, voici quelques exemples de clients de R IFSSSO PLU S : hôpitaux, centres d'accès aux soins communautaires, centres de santé communautaire, services de santé publique des municipalités, sociétés d'aide àl'enfance, institutions de formation, gouvernements provinciaux et gouvernement fédéral et secteur privé : banques, compagnies d'assurance, etc.

\section{En résumé}

Le R IFSSSO est le résultat d'une activité entre individus visant un but commun bien défini : la santé en français en milieu minoritaire.

- N ous avons bâti sur nos droits.

- N ous avons solidifié des partenariats multiples.

- $N$ ous agissons de manière économique.

- N ous exploitons les communications d'avant-garde.

- N ouspossédonsune expertise unique en milieux minoritaires que nous partageons avec nos collègues au- delà de la province.

R IFSSSO - PLU S, un service unique, de qualité, multidisciplinaire et flexible! 